\title{
Effects of hot water treatments on dormant grapevine propagation materials used for grafted vine production
}

\author{
Oguzhan Soltekin ${ }^{1, \mathrm{a}}$ and Ahmet Altindisli ${ }^{2}$ \\ ${ }^{1}$ Manisa Viticultural Research Institute, Ataturk St., Yunusemre, PO Box 12, Manisa, Turkey \\ ${ }^{2}$ Ege University, Faculty of Agriculture, Department of Horticulture, Izmir, Turkey
}

\begin{abstract}
Agrobacterium vitis is responsible for the crown gall disease of grapevine which breaks the grapevine trunk vascular system. Nutrient flow is prevented by crown gall and it leads to weak growth and death of the plants. It can be destructive disease often encountered in vineyards and it can be spread in cuttings for propagation. Thermotherapy treatment is an alternative method for eradicating A. vitis from grapevine cuttings but effects of thermotherapy treatments on dormant vine tissue, bud vitality, rooting and shooting of the propagation materials are not yet fully understood. In this research, it is aimed to determine the effects of thermotherapy treatment (Hot water treatment) on callus formation (at the basal part and grafting point), grafted vine quality (shoot length, shoot width, root number, shooting and rooting development, fresh and dry weight of shoots and roots) and final take in the grafted vine production. Experiment was conducted in the nursery of Manisa Viticultural Research Institute. Rootstocks (Kober 5BB, Couderc 1613 and 41B) and scions (Sultan 7 and Manisa sultan1) were hot-water treated at $50^{\circ} \mathrm{C}$ for 30 minutes which is the most common technique against Agrobacterium vitis. After thermotherapy treatment, all rootstocks were grafted with Sultan 7 and Manisa sultanivarieties. They were kept for 22 days in callusing room for callus development and then they were planted in polyethlyene bags for rooting. At the end of the study, significant treatment x rootstock interaction were observed for the final take of Sultan 7 variety. Thermotherapy treated of $1613 \mathrm{C} / \mathrm{Sultan} 7$ combinations had more final take than the control (untreated) group. For instance, hot water treated cuttings of $1613 \mathrm{C} /$ Sultan 7 combinations had $75 \%$ final take while the control group had the $70 \%$. Also there were not observed any adverse effects of HWT on bud and tissue vitality.
\end{abstract}

\section{Introduction}

Viticulture constitutes an important part of agricultural production in the world and it is common between $20-52^{\circ}$ latitudes in the Northern Hemisphere and $20-40^{\circ}$ latitudes for Southern Hemisphere. Turkey, which has a strong viticultural potential, is one of the important gene centers of the vine. Today, 74 million tons of world grape yield (production) that is 4.1 million tons yielded in Turkey. In addition, Turkey ranks 5th in the world with 467.093 ha grape harvested area [1] and that the grape yield per hectare is about 8.9 tons. It can be observed that this value is considerably less than the advanced countries of viticulture. This situation is due to various negativities encountered during the period planting to the marketing. Especially, some of the pests and diseases can affect adversely the grape yield per unit area in our country.

Crown gall, caused by the soil-borne bacterium (Agrobacterium vitis), is also the most important disease in our country's vineyards. The most remarkable signs are galls and cracks that appear on grapevine trunks [2]. Galls are more common on the lower trunk but they can develop in canes and at graft unions. Crown gall can kill young vines, reduce growing potential and require establishment of new trunks [3].

There are not any effective chemical treatments for grape crown gall control yet [4]. Therefore its control

a e-mail: oguz.soltekin@tarim.gov.tr focuses on prevention of injuries and using of pathogen free propagation materials [5].

In our country, grafted vine producers may select the infected cuttings in the vineyard for grafting process without being aware of it. Therefore the possibility of using infected propagation materials with Agrobacterium vitis while establishment of the vineyards, is one of the enhancing dissemination and transmission of disease. Furthermore if Agrobacterium vitis identify in herbal products, it is among the organisms subject to quarantine which is prohibited to circulate in our country. Therefore it is determined that A. vitis has an extremely important precaution for our country, according to the Regulation on "Plant passport system and registration of operators" published in the official gazette dated 2nd January 2011, numbered 27813 [6].

Hot water treatment (Thermotherapy) is an efficient, environmentaly safe and commercially viable method for sterilization of Agrobacterium vitis [7]. The most common hot water treatment for dormant propagation materials (rootstocks and vine cuttings) is submerging them in a water bath at $50^{\circ} \mathrm{C}$ for $30 \mathrm{~min}[3,7,8]$. However the most common problems in HWT studies are generally related its affects on bud and tissue vitality, growth in the nursery and the effects on grafting of vines for different varieties [3,9-13].

In this study, it was aimed to determine the effects of hot-water treatment (thermotherapy) on propagation 
materials used for grafted vine production. In this way, new registered grape varieties (Sultan 7 and Manisa sultanı) were used as plant materials and it was observed responses of these varieties to the thermotherapy treatments.

\section{Materials and methods}

\subsection{Plant material}

This research was carried out in the nursery of Viticultural Research Institute in Manisa province. Five rootstock cultivars Kober 5BB, Couderc 1613, 41B and two scion cultivars Sultan 7, Manisa Sultaniwere used as plant material. Sultan 7 is a seedless variety and it is the most common raisin variety in Turkey. It matures in second half of the August. It has high drying efficiency and yield potential. Manisa Sultaniis a seedless variety growing for primarily table grape. Berries are elliptic, greenyellow color. It matures in second half of the August. All these propagation materials were collected in the dormant season (November and February) from the vineyards of the Institute. Then they were placed in cold storage at $2-4^{\circ} \mathrm{C}$ until grafting. Sultan 7 and Manisa Sultanırape varieties are newly registered varieties. Therefore, in this research, it was observed responses of these varieties to the hot water treatment (HWT) first time.

\subsection{Methods}

In the research, all propagation materials (rootstocks and scions) were kept in the room temperature overnight before the HWT. Then they were immersed in hot-water at $50^{\circ} \mathrm{C}$ for 30 minutes which is the most common technique against Agrobacterium vitis. Hot water tank had $1000 \mathrm{~L}$ water capacity with a recirculation pump and temperature was monitored via probes during the treatment process. After HWT, all propagation materials were left to drying at room temperature. Also there were not any hot water treatments for control group.

One bud scions of the Sultan 7 and Manisa sultanigrape varieties were omega shaped bench-grafted onto three rootstocks $[14,15]$. Grafted cuttings were dipped into paraffin wax $\left(55-60^{\circ} \mathrm{C}\right)$ to cover the grafting point and placed in plastic boxes filled up with sawdust and coarse chip (3:1). All boxes were kept in callusing room (25-27 $\mathrm{C}, 90-95 \%$ humidity) for three weeks and then transferred to the outdoor conditions for adaptation in two days. When the callus formation was completed in the callusing room, rooting rates $(\%)$ and sprouting rates (\%) were determined. Then, callusing level at the grafting point and at the basal part were measured according to the $0-4$ scale (0-no callus development, 1- weak callus development, 2- crescent shaped callus development, 3- partially interrupted callus development, 4- complete callus development).

After these measurements, grafted cuttings were planted in polyethlyene bags for rooting over 6-7 weeks at $25-27^{\circ} \mathrm{C}, 70-75 \%$ humidity in greenhouse. At the end of the this growing period in greenhouse, following parameters were measured; Shooting and rooting development level (0-4 scale), shoot length $(\mathrm{cm})$, shoot width $(\mathrm{mm})$, root number, fresh and dry weight of shoots and roots $(\mathrm{g})$ and final take $(\%)$ were measured. All parameters were examined to determine the effects of thermotherapy treatments on propagation materials (rootstocks and scions) for grafted vine production.

The experimental design was randomized plots with three replications and there were 30 cuttings in each replication. Analysis of variance is implemented to research data by using SPSS statistical analysis software package on computer, and in order to determine the differences among averages, LSD test is implemented.

\section{Results and discussion}

According to the measurements of after callus formation in the callusing room, effect of hot water treatment on rooting and sprouting rates was found non-significant. However hot water treated cuttings of all rootstocks x Manisa Sultanicombinations had higher values than control group in terms of rooting rates. Also the highest values were obtained from the hot water treated (64.4\%) and untreated $(56.7 \%)$ 1613C x Manisa Sultanicombinations respectively (Table 1 and Table 2). Besides that [16] reported similar results in their study. For instance, they observed different sprouting ratios during the callusing development for the all rootstock $\mathrm{x}$ variety combinations. On the other hand the highest rooting ratio was obtained from the $1613 \mathrm{C} \times$ variety combinations $(54.6 \%)$ in their study.

The Table 3 and Table 4 show that effects of HWT on callusing level at the basal part and grafting point, respectively. Treatment $\mathrm{x}$ rootstocks interactions for the Sultan 7 variety were found statistically significant in terms of callusing level at the basal part. The highest value (2.65) was observed in hot water treated vines of $41 \mathrm{~B} /$ Sultan 7 combination. Also [17] reported that thermotherapy treatments stimulated callus formation at the basal part in most cases.

When measuring the callus formation at the grafting point, the lowest level were observed in hot water treated vines of $5 \mathrm{BB} / \mathrm{Sultan} 7$ combination (2.30). On the other hand the highest values were observed in $1613 \mathrm{C} / \mathrm{Sultan} 7$ combinations. As can be seen from the results, callus formation was affected adversely by hot water treatment on 5BB/Sultan 7 and 5BB/ Manisa Sultanicombinations. However other combinations were placed in the highest level group statistically (Table 4). When the callus formation was considered as one of the important criteria determining the success in the production of grafted vines [18], 41B and $1613 \mathrm{C}$ rootstocks come into prominence for the both varieties. Hot water treated cuttings of 41B/Manisa Sultanıcombinations was found $6.5 \%$ more callus formation than untreated group. In a study hot water treated and grafted cuttings were found 5-10\% better callus formation than the control cuttings [3].

Significant treatment $\mathrm{x}$ rootstock interactions were observed for the Sultan 7 variety in terms of shooting and rooting development levels (Table 5 and Table 6). Hot water treated vines of $1613 \mathrm{C} / \mathrm{Sultan} 7$ and $41 \mathrm{~B} / \mathrm{Sultan} 7$ combinations had higher values than each control groups for the both shooting and rooting development levels. As can be seen from the results of all rootstocks $\mathrm{x}$ Sultan 7 combinations, hot water treatments induced more shooting and rooting development except 5BB/Sultan 7 combination. These findings was found parallel with the previous studies $[11,17,19]$. 
Table 1. Effects of HWT on rooting rates (\%).

\begin{tabular}{|c|c|c|c|c|c|c|c|}
\hline \multicolumn{4}{|c|}{ Manisa Sultant } & \multicolumn{4}{|c|}{ Sultan 7} \\
\hline Rootstocks & Control & HWT & Rootstock Mean & Rootstocks & Control & HWT & Rootstock Mean \\
\hline $5 \mathrm{BB}$ & 32.400 & 33.333 & $32,867 \mathrm{~b}$ & $5 \mathrm{BB}$ & 51.297 & 34.167 & 42.732 \\
\hline $41 \mathrm{~B}$ & 43.380 & 49.417 & $46,398 \mathrm{ab}$ & $41 \mathrm{~B}$ & 41.733 & 54.373 & 48.053 \\
\hline $1613 \mathrm{C}$ & 56.783 & 64.430 & $60,607 a$ & $1613 \mathrm{C}$ & 61.03 & 52.943 & 56.987 \\
\hline Treatment Mean & 44.188 & 49.060 & & Treatment Mean & 51.353 & 47.161 & \\
\hline \multicolumn{4}{|c|}{ LSD Treatment: $\mathrm{ns}$} & \multicolumn{4}{|c|}{ LSD Treatment: $\mathrm{ns}$} \\
\hline \multicolumn{4}{|c|}{ LSD Rootstock: $15,219 * *$} & \multicolumn{4}{|c|}{ LSD Rootstock: ns } \\
\hline \multicolumn{4}{|c|}{ LSD Treatment x Rootstock: ns } & \multicolumn{4}{|c|}{ LSD Treatment x Rootstock: ns } \\
\hline
\end{tabular}

Table 2. Effects of HWT on sprouting rates (\%).

\begin{tabular}{|c|c|c|c|c|c|c|c|}
\hline \multicolumn{4}{|c|}{ Manisa Sultanı } & \multicolumn{4}{|c|}{ Sultan 7} \\
\hline Rootstocks & Control & HWT & Rootstock Mean & Rootstocks & Control & HWT & Rootstock Mean \\
\hline $5 \mathrm{BB}$ & 30.070 & 30.640 & 30.355 & $5 \mathrm{BB}$ & 40.977 & 36.113 & 38.545 \\
\hline $41 \mathrm{~B}$ & 35.417 & 35.417 & 35.417 & $41 \mathrm{~B}$ & 31.25 & 41.667 & 36.458 \\
\hline $1613 \mathrm{C}$ & 37.693 & 37.190 & 37.442 & $1613 \mathrm{C}$ & 37.447 & 28.677 & 33.062 \\
\hline Treatment Mean & 34.393 & 34.416 & & Treatment Mean & 36.558 & 35.486 & \\
\hline \multicolumn{4}{|c|}{ LSD Treatment: $\mathrm{ns}$} & \multicolumn{4}{|c|}{ LSD Treatment: $\mathrm{ns}$} \\
\hline \multicolumn{4}{|c|}{ LSD Rootstock: ns } & \multicolumn{4}{|c|}{ LSD Rootstock: ns } \\
\hline \multicolumn{4}{|c|}{ LSD Treatment x Rootstock: ns } & \multicolumn{4}{|c|}{ LSD Treatment x Rootstock: ns } \\
\hline
\end{tabular}

Table 3. Callusing level at the basal part (0-4).

\begin{tabular}{|c|c|c|c|c|c|c|c|}
\hline \multicolumn{4}{|c|}{ Manisa Sultant } & \multicolumn{4}{|c|}{ Sultan 7} \\
\hline Rootstocks & Control & HWT & Rootstock Mean & Rootstocks & Control & HWT & Rootstock Mean \\
\hline 5BB & 2.70 & 1.10 & $1,900 \mathrm{a}$ & 5BB & $2,393 a$ & $0,973 \mathrm{~b}$ & $1,683 \mathrm{~b}$ \\
\hline 41B & 2.97 & 1.84 & $2,407 \mathrm{a}$ & 41B & $2,580 \mathrm{a}$ & $2,650 \mathrm{a}$ & $2,615 \mathrm{a}$ \\
\hline $1613 \mathrm{C}$ & 0.59 & 0.59 & $0,593 b$ & $1613 \mathrm{C}$ & $0,647 b$ & $0,753 b$ & $0,700 \mathrm{c}$ \\
\hline Treatment Mean & $2,088 \mathrm{a}$ & $1,179 \mathrm{~b}$ & & Treatment Mean & $1,873 \mathrm{a}$ & $1,459 \mathrm{~b}$ & \\
\hline \multicolumn{4}{|c|}{ LSD Treatment: $0,835^{* *}$} & \multicolumn{4}{|c|}{ LSD Treatment: $0,399 *$} \\
\hline \multicolumn{4}{|c|}{ LSD Rootstock: $1,022 * *$} & \multicolumn{4}{|c|}{ LSD Rootstock: $0,685^{* *}$} \\
\hline \multicolumn{4}{|c|}{ LSD Treatment x Rootstock: ns } & \multicolumn{4}{|c|}{ LSD Treatment x Rootstock: $0,969 * *$} \\
\hline
\end{tabular}

Table 4. Callusing level at the grafting point (0-4).

\begin{tabular}{|c|c|c|c|c|c|c|c|}
\hline \multicolumn{4}{|c|}{ Manisa Sultanı } & \multicolumn{4}{|c|}{ Sultan 7} \\
\hline Rootstocks & Control & HWT & Rootstock Mean & Rootstocks & Control & HWT & Rootstock Mean \\
\hline $5 \mathrm{BB}$ & $3,777 \mathrm{a}$ & $3,333 b$ & $3,555 \mathrm{~b}$ & $5 \mathrm{BB}$ & $3,807 \mathrm{a}$ & $2,303 b$ & $3,055 \mathrm{c}$ \\
\hline $41 \mathrm{~B}$ & $3,697 a$ & $3,940 \mathrm{a}$ & $3,818 \mathrm{a}$ & $41 \mathrm{~B}$ & $3,593 \mathrm{a}$ & $3,623 a$ & $3,608 \mathrm{~b}$ \\
\hline $1613 \mathrm{C}$ & $3,867 \mathrm{a}$ & $3,867 \mathrm{a}$ & $3,867 \mathrm{a}$ & $1613 \mathrm{C}$ & $4,000 \mathrm{a}$ & $3,960 \mathrm{a}$ & $3,980 \mathrm{a}$ \\
\hline Treatment Mean & 3.780 & 3.713 & & Treatment Mean & $3,800 \mathrm{a}$ & $3,296 b$ & \\
\hline \multicolumn{4}{|c|}{ LSD Treatment: $\mathrm{ns}$} & \multicolumn{4}{|c|}{ LSD Treatment: $0,242 * *$} \\
\hline \multicolumn{4}{|c|}{ LSD Rootstock: $0,206^{*}$} & \multicolumn{4}{|c|}{ LSD Rootstock: $0,296^{* *}$} \\
\hline \multicolumn{4}{|c|}{ LSD Treatment x Rootstock: $0,291^{*}$} & \multicolumn{4}{|c|}{ LSD Treatment $\mathrm{x}$ Rootstock: $0,419 * *$} \\
\hline
\end{tabular}

Table 5. Effects of HWT on shooting development level (0-4).

\begin{tabular}{|c|c|c|c|c|c|c|c|}
\hline \multicolumn{4}{|c|}{ Manisa Sultanı } & \multicolumn{4}{|c|}{ Sultan 7} \\
\hline Rootstocks & Control & HWT & Rootstock Mean & Rootstocks & Control & HWT & Rootstock Mean \\
\hline $5 \mathrm{BB}$ & 1.900 & 1.633 & $1.767 \mathrm{~b}$ & $5 \mathrm{BB}$ & $2.657 \mathrm{ab}$ & $1.733 \mathrm{~d}$ & $2.195 b$ \\
\hline 41B & 2.410 & 2.247 & $2.328 \mathrm{ab}$ & 41B & $1.913 \mathrm{~cd}$ & $2.243 \mathrm{bcd}$ & $2.078 \mathrm{~b}$ \\
\hline $1613 \mathrm{C}$ & 2.330 & 3.020 & $2.677 \mathrm{a}$ & $1613 \mathrm{C}$ & $2.553 a b c$ & $3.007 \mathrm{a}$ & $2.780 \mathrm{a}$ \\
\hline Treatment Mean & 2.214 & 2.300 & & Treatment Mean & 2.374 & 2.328 & \\
\hline \multicolumn{4}{|c|}{ LSD Treatment: ns } & \multicolumn{4}{|c|}{ LSD Treatment: $\mathrm{ns}$} \\
\hline \multicolumn{4}{|c|}{ LSD Rootstock: $0.575^{*}$} & \multicolumn{4}{|c|}{ LSD Rootstock: $0.496^{* *}$} \\
\hline \multicolumn{4}{|c|}{ LSD Treatment x Rootstock: ns } & \multicolumn{4}{|c|}{ LSD Treatment $\mathrm{x}$ Rootstock: $0.702 * *$} \\
\hline
\end{tabular}


Table 6. Effects of HWT on rooting development level (0-4).

\begin{tabular}{|c|c|c|c|c|c|c|c|}
\hline \multicolumn{4}{|c|}{ Manisa Sultanı } & \multicolumn{4}{|c|}{ Sultan 7} \\
\hline Rootstocks & Control & HWT & Rootstock Mean & Rootstocks & Control & HWT & Rootstock Mean \\
\hline $5 \mathrm{BB}$ & 1.833 & 1.750 & $1,792 \mathrm{~b}$ & $5 \mathrm{BB}$ & $2,500 \mathrm{~b}$ & $1,500 \mathrm{c}$ & $2,000 \mathrm{~b}$ \\
\hline 41B & 2.833 & 2.333 & $2,583 \mathrm{a}$ & $41 \mathrm{~B}$ & $1,500 \mathrm{c}$ & $3,000 \mathrm{ab}$ & $2,250 \mathrm{~b}$ \\
\hline $1613 \mathrm{C}$ & 2.500 & 2.500 & $2,500 \mathrm{a}$ & $1613 \mathrm{C}$ & $2,830 \mathrm{~b}$ & $3,830 \mathrm{a}$ & $3,333 \mathrm{a}$ \\
\hline Treatment Mean & 2.389 & 2.194 & & Treatment Mean & $2,278 b$ & $2,778 \mathrm{a}$ & \\
\hline \multicolumn{4}{|c|}{ LSD Treatment: $\mathrm{ns}$} & \multicolumn{4}{|c|}{ LSD Treatment: $0,401^{*}$} \\
\hline \multicolumn{4}{|c|}{ LSD Rootstock: $0.529 *$} & \multicolumn{4}{|c|}{ LSD Rootstock: $0,689 * *$} \\
\hline \multicolumn{4}{|c|}{ LSD Treatment $\mathrm{x}$ Rootstock: $\mathrm{ns}$} & \multicolumn{4}{|c|}{ LSD Treatment $\mathrm{x}$ Rootstock: $0,975^{* *}$} \\
\hline
\end{tabular}

Table 7. Effects of HWT on shoot length (cm).

\begin{tabular}{|l|c|c|c|l|c|c|c|}
\hline \multicolumn{5}{|c|}{ Manisa Sultani } & \multicolumn{5}{|c|}{ Sultan 7 } \\
\hline Rootstocks & Control & HWT & Rootstock Mean & Rootstocks & Control & HWT & Rootstock Mean \\
\hline 5BB & 9.897 & 7.667 & $8,782 \mathrm{~b}$ & $5 \mathrm{BB}$ & $14,783 \mathrm{a}$ & $9,933 \mathrm{~b}$ & $12,358 \mathrm{ab}$ \\
\hline 41B & 13.210 & 12.333 & $12,772 \mathrm{ab}$ & $41 \mathrm{~B}$ & $9,440 \mathrm{~b}$ & $10,540 \mathrm{~b}$ & $9,990 \mathrm{~b}$ \\
\hline 1613C & 12.610 & 17.903 & $15,257 \mathrm{a}$ & $1613 \mathrm{C}$ & $13,200 \mathrm{ab}$ & $15,887 \mathrm{a}$ & $14,543 \mathrm{a}$ \\
\hline Treatment Mean & 11.906 & 12.634 & & Treatment Mean & 12.474 & 12.120 & \\
\hline
\end{tabular}

Table 8. Effects of HWT on shoot width (mm).

\begin{tabular}{|c|c|c|c|c|c|c|c|}
\hline \multicolumn{4}{|c|}{ Manisa Sultanı } & \multicolumn{4}{|c|}{ Sultan 7} \\
\hline Rootstocks & Control & HWT & Rootstock Mean & Rootstocks & Control & HWT & Rootstock Mean \\
\hline $5 \mathrm{BB}$ & 1.830 & 1.507 & 1.668 & $5 \mathrm{BB}$ & 1.973 & 1.620 & 1.797 \\
\hline $41 \mathrm{~B}$ & 2.010 & 1.643 & 1.827 & $41 \mathrm{~B}$ & 2.143 & 5.330 & 3.737 \\
\hline $1613 \mathrm{C}$ & 1.950 & 2.187 & 2.068 & $1613 \mathrm{C}$ & 1.847 & 1.920 & 1.883 \\
\hline Treatment Mean & 1.930 & 1.779 & & Treatment Mean & 1.988 & 2.957 & \\
\hline \multicolumn{4}{|c|}{ LSD Treatment: $\mathrm{ns}$} & \multicolumn{4}{|c|}{ LSD Treatment: $\mathrm{ns}$} \\
\hline \multicolumn{4}{|c|}{ LSD Rootstock: ns } & \multicolumn{4}{|c|}{ LSD Rootstock: ns } \\
\hline \multicolumn{4}{|c|}{ LSD Treatment x Rootstock: ns } & \multicolumn{4}{|c|}{ LSD Treatment $x$ Rootstock: ns } \\
\hline
\end{tabular}

Table 9. Effects of HWT on root number.

\begin{tabular}{|c|c|c|c|c|c|c|c|}
\hline \multicolumn{4}{|c|}{ Manisa Sultanı } & \multicolumn{4}{|c|}{ Sultan 7} \\
\hline Rootstocks & Control & HWT & Rootstock Mean & Rootstocks & Control & HWT & Rootstock Mean \\
\hline $5 \mathrm{BB}$ & $12,083 \mathrm{c}$ & $8,500 \mathrm{c}$ & $10,292 \mathrm{~b}$ & $5 \mathrm{BB}$ & $14,000 \mathrm{~cd}$ & $11,183 \mathrm{~cd}$ & $12,592 \mathrm{~b}$ \\
\hline $41 \mathrm{~B}$ & $27,333 \mathrm{ab}$ & $13,000 \mathrm{bc}$ & $20,167 b$ & $41 \mathrm{~B}$ & $8,000 \mathrm{~d}$ & $18,667 \mathrm{c}$ & $13,333 b$ \\
\hline $1613 \mathrm{C}$ & $27,333 \mathrm{ab}$ & $41,667 \mathrm{a}$ & $34,500 \mathrm{a}$ & $1613 \mathrm{C}$ & $36,000 b$ & $49,667 \mathrm{a}$ & $42,833 a$ \\
\hline Treatment Mean & 22.250 & 21.056 & & Treatment Mean & $19,333 b$ & $26,506 a$ & \\
\hline \multicolumn{4}{|c|}{ LSD Treatment: ns } & \multicolumn{4}{|c|}{ LSD Treatment: 5,099** } \\
\hline \multicolumn{4}{|c|}{ LSD Rootstock: $10,209 * *$} & \multicolumn{4}{|c|}{ LSD Rootstock: $6,245 * *$} \\
\hline \multicolumn{4}{|c|}{ LSD Treatment x Rootstock: $14,438 * *$} & \multicolumn{4}{|c|}{ LSD Treatment x Rootstock: $8,832 * *$} \\
\hline
\end{tabular}

The Table 7 shows that effects of hot water treatments on shoot length while the Table 8 shows the effects of hot water treatments on shoot width. Significant treatment $\mathrm{x}$ rootstock interactions were observed for the shoot length of Sultan 7 variety. The highest values were observed in hot water treated vines of $1613 \mathrm{C} / \mathrm{Sultan} 7$ $(15.89 \mathrm{~cm})$ combinations. Also hot water treated cuttings was found $20.3 \%$ and $11.7 \%$ more shoot length than the untreated ones in $1613 \mathrm{C} /$ Sultan 7 and $41 \mathrm{~B} / \mathrm{Sultan}$ 7 combinations respectively. The effect of hot water treatment on shoot width was statistically non-significant for both varieties. However the highest value was observed in hot water treated vines of $41 \mathrm{~B} / \mathrm{Sultan} 7(5.33 \mathrm{~mm})$ and 1613C/Manisa Sultanı $(2.19 \mathrm{~mm})$ combinations. Also in previous studies, it was observed that the effects of thermotherapy treatments were varied in terms of shoot length and shoot width on different rootstock $\mathrm{x}$ variety combinations $[13,20]$. In a study carried out by [3], hot water treated and grafted vines of K51-40/Zante Currant and Ramsey/Zante Currant combinations had longer shoots than the control group.

For both varieties, significant treatment $\mathrm{x}$ rootstock interaction were observed in terms of root number. Hot water treated vines of 1613C/Manisa sultaniand 


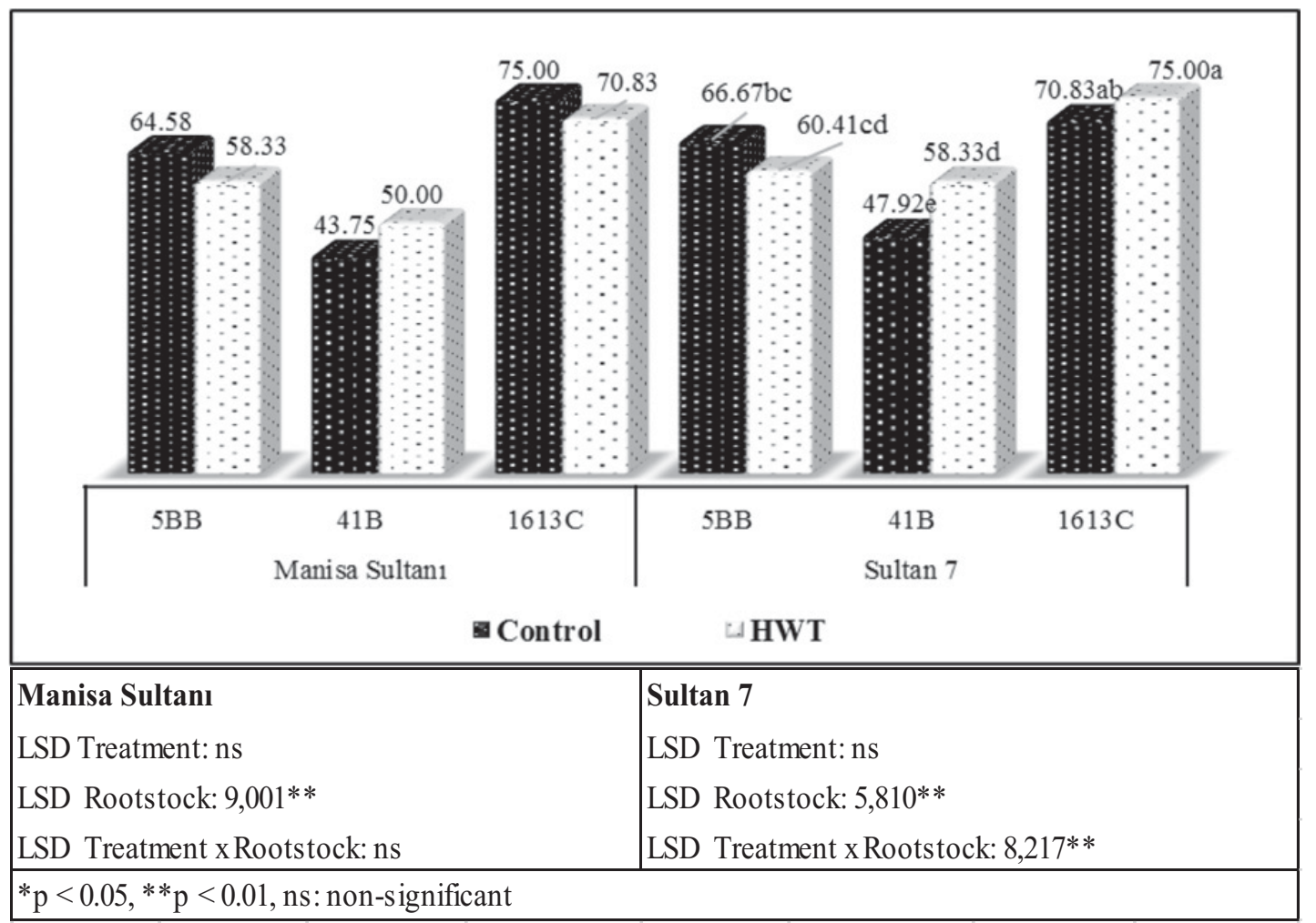

Figure 1. Effects of HWT on Final Take value (\%).

Table 10. Effects of HWT on fresh weight of shoots (g).

\begin{tabular}{|c|c|c|c|c|c|c|c|}
\hline \multicolumn{4}{|c|}{ Manisa Sultanı } & \multicolumn{4}{|c|}{ Sultan 7} \\
\hline Rootstocks & Control & HWT & Rootstock Mean & Rootstocks & Control & HWT & Rootstock Mean \\
\hline $5 \mathrm{BB}$ & $45,710 \mathrm{c}$ & $44,253 \mathrm{~cd}$ & $44,982 \mathrm{~b}$ & $5 \mathrm{BB}$ & $51,143 b$ & $37,330 \mathrm{c}$ & $44,237 b$ \\
\hline $41 \mathrm{~B}$ & $42,533 d$ & $46,363 c$ & $44,448 b$ & $41 \mathrm{~B}$ & $39,050 \mathrm{c}$ & $45,143 b c$ & $42,097 b$ \\
\hline $1613 \mathrm{C}$ & $54,640 \mathrm{~b}$ & $57,590 \mathrm{a}$ & $56,115 a$ & $1613 \mathrm{C}$ & $51,877 \mathrm{~b}$ & $64,267 \mathrm{a}$ & $58,072 \mathrm{a}$ \\
\hline Treatment Mean & $47,628 b$ & $49,402 \mathrm{a}$ & & Treatment Mean & 47.357 & 48.913 & \\
\hline \multicolumn{4}{|c|}{ LSD Treatment: $1,599 *$} & \multicolumn{4}{|c|}{ LSD Treatment: $\mathrm{ns}$} \\
\hline \multicolumn{4}{|c|}{ LSD Rootstock: $2,747 * *$} & \multicolumn{4}{|c|}{ LSD Rootstock: $6,132^{* *}$} \\
\hline \multicolumn{4}{|c|}{ LSD Treatment x Rootstock: $2,770^{*}$} & \multicolumn{4}{|c|}{ LSD Treatment $\mathrm{x}$ Rootstock: $8,672^{* *}$} \\
\hline
\end{tabular}

Table 11. Effects of HWT on dry weight of shoots (g).

\begin{tabular}{|c|c|c|c|c|c|c|c|}
\hline \multicolumn{4}{|c|}{ Manisa Sultant } & \multicolumn{4}{|c|}{ Sultan 7} \\
\hline Rootstocks & Control & HWT & Rootstock Mean & Rootstocks & Control & HWT & Rootstock Mean \\
\hline $5 \mathrm{BB}$ & 10.050 & 10.620 & $10,335 b$ & $5 \mathrm{BB}$ & $11,077 \mathrm{~b}$ & $11,190 \mathrm{~b}$ & 11.133 \\
\hline $41 \mathrm{~B}$ & 11.400 & 11.013 & $11,207 \mathrm{~b}$ & $41 \mathrm{~B}$ & $10,273 b$ & $12,870 \mathrm{a}$ & 11.572 \\
\hline $1613 \mathrm{C}$ & 14.220 & 13.890 & $14,055 \mathrm{a}$ & $1613 \mathrm{C}$ & $11,630 \mathrm{ab}$ & $11,797 \mathrm{ab}$ & 11.713 \\
\hline Treatment Mean & 11.890 & 11.841 & & Treatment Mean & $10,993 b$ & $11,952 \mathrm{a}$ & \\
\hline \multicolumn{4}{|c|}{ LSD Treatment: $\mathrm{ns}$} & \multicolumn{4}{|c|}{ LSD Treatment: $0.943^{* *}$} \\
\hline \multicolumn{4}{|c|}{ LSD Rootstock: $0,972 * *$} & \multicolumn{4}{|c|}{ LSD Rootstock: ns } \\
\hline \multicolumn{4}{|c|}{ LSD Treatment $\mathrm{x}$ Rootstock: $\mathrm{ns}$} & \multicolumn{4}{|c|}{ LSD Treatment $\mathrm{x}$ Rootstock: $1.633^{* *}$} \\
\hline
\end{tabular}

1613C/Sultan7 combinations had higher values compare to the control vines. Root number of grafted vines for the hot water treated 1613C/Manisa sultanicombination was 41.67 while $1613 \mathrm{C} /$ Sultan 7 combination was 49.67 (Table 9). Also it was observed more root numbers with the hot water treatments in 41B/Sulltan7, 1613C/Sultan7 and 1613C/Manisa sultanicombinations. In a study conducted by [13], there was an increment in the root numbers with the hot water treatments.
According to the measurements of final take, it was found that significant treatment $\mathrm{x}$ rootstock interaction for the Sultan 7 variety (Fig. 1). Hot water treated group of $41 \mathrm{~B} /$ Sultan 7 and $1613 \mathrm{C} /$ Sultan 7 combinations had $21.7 \%$ and $5.9 \%$ more final take than the control group. On the other hand hot water treated vines of $5 \mathrm{BB} / \mathrm{Sultan}$ 7 combinations had $9.4 \%$ lower final take compare to the control group. Generally hot water treatment affected the final take positively in the rootstocks $x$ Sultan 7 
Table 12. Effects of HWT on fresh weight of roots (g).

\begin{tabular}{|c|c|c|c|c|c|c|c|}
\hline \multicolumn{4}{|c|}{ Manisa Sultanı } & \multicolumn{4}{|c|}{ Sultan 7} \\
\hline Rootstocks & Control & HWT & Rootstock Mean & Rootstocks & Control & HWT & Rootstock Mean \\
\hline $5 \mathrm{BB}$ & 57.823 & 55.587 & $56,705 b$ & 5BB & $70,187 \mathrm{a}$ & $63,340 \mathrm{ab}$ & $66,763 \mathrm{a}$ \\
\hline 41B & 64.520 & 64.663 & $64,592 \mathrm{a}$ & $41 \mathrm{~B}$ & $42,430 \mathrm{e}$ & $50,710 \mathrm{~d}$ & $46,570 \mathrm{c}$ \\
\hline $1613 \mathrm{C}$ & 67.047 & 62.157 & $64,602 a$ & $1613 \mathrm{C}$ & $52,033 \mathrm{~cd}$ & $59,733 b c$ & $55,883 b$ \\
\hline Treatment Mean & 63.130 & 60.802 & & Treatment Mean & 54.883 & 57.928 & \\
\hline \multicolumn{4}{|c|}{ LSD Treatment: $\mathrm{ns}$} & \multicolumn{4}{|c|}{ LSD Treatment: $\mathrm{ns}$} \\
\hline \multicolumn{4}{|c|}{ LSD Rootstock: $4,172^{* *}$} & \multicolumn{4}{|c|}{ LSD Rootstock: 5,607** } \\
\hline \multicolumn{4}{|c|}{ LSD Treatment $\mathrm{x}$ Rootstock: ns } & \multicolumn{4}{|c|}{ LSD Treatment $\mathrm{x}$ Rootstock: 7,930** } \\
\hline
\end{tabular}

Table 13. Effects of HWT on dry weight of roots (g).

\begin{tabular}{|c|c|c|c|c|c|c|c|}
\hline \multicolumn{4}{|c|}{ Manisa Sultanı } & \multicolumn{4}{|c|}{ Sultan 7} \\
\hline Rootstocks & Control & HWT & Rootstock Mean & Rootstocks & Control & HWT & Rootstock Mean \\
\hline $5 \mathrm{BB}$ & $6,587 \mathrm{c}$ & $8,720 \mathrm{bc}$ & $7,653 \mathrm{c}$ & $5 \mathrm{BB}$ & $8,237 \mathrm{~b}$ & $9,300 \mathrm{ab}$ & $8,237 \mathrm{a}$ \\
\hline $41 \mathrm{~B}$ & $9,813 \mathrm{~b}$ & $10,247 \mathrm{~b}$ & $10,030 \mathrm{~b}$ & $41 \mathrm{~B}$ & $4,063 \mathrm{~d}$ & $10,927 \mathrm{a}$ & $4,063 \mathrm{c}$ \\
\hline $1613 \mathrm{C}$ & $13,140 \mathrm{a}$ & $10,147 \mathrm{~b}$ & $11,643 a$ & $1613 \mathrm{C}$ & $6,347 \mathrm{c}$ & $9,697 \mathrm{ab}$ & $6,347 \mathrm{~b}$ \\
\hline Treatment Mean & 9.847 & 9.704 & & Treatment Mean & $6,216 \mathrm{~b}$ & $9,974 \mathrm{a}$ & \\
\hline \multicolumn{4}{|c|}{ LSD Treatment: $\mathrm{ns}$} & \multicolumn{4}{|c|}{ LSD Treatment: $1,089 * *$} \\
\hline \multicolumn{4}{|c|}{ LSD Rootstock: $1,568 * *$} & \multicolumn{4}{|c|}{ LSD Rootstock: $0,951^{*}$} \\
\hline \multicolumn{4}{|c|}{ LSD Treatment $\mathrm{x}$ Rootstock: $2,218^{* *}$} & \multicolumn{4}{|c|}{ LSD Treatment $\mathrm{x}$ Rootstock: $1,885^{* *}$} \\
\hline
\end{tabular}

combinations. However it was observed that $5 \mathrm{BB} /$ variety combination was more sensitive than the others and this combination was affected adversely by thermotherapy treatment. Additionally it was observed that thermotherapy treatments effects can be changed by the varieties. These findings were similar to the previous studies $[8,11,13,19,21]$.

According to the fresh shoot weight values, significant treatment $\mathrm{x}$ rootstock interaction were observed for the Manisa Sultaniand Sultan 7 varieties (Table 10). Hot water treated vines of all combinations, except 5BB/Manisa sultanıand 5BB/Sultan 7 combinations had higher fresh shoot weight values compare to the control group. Additionally hot water treated vines of 1613C/Manisa Sultaniand 1613C/Sultan 7 combinations had the highest fresh shoot weight values for each variety, $57.59 \mathrm{~g}$ and $64.27 \mathrm{~g}$ respectively. Also significant treatment x rootstock interaction was found for the Sultan 7 variety in terms of dry shoot weight (Table 11). The highest and lowest value was obtained from hot water treated $(12.87 \mathrm{~g})$ and untreated $(10.27 \mathrm{~g})$ vines of $41 \mathrm{~B} / \mathrm{Sultan} 7$ combination respectively.

The Table 12 shows that effect of HWT on fresh weight of roots. In Sultan 7 variety, it was determined that significant treatment $\mathrm{x}$ rootstock interaction. The highest value was observed in hot water treated vine of $1613 \mathrm{C} / \mathrm{Sultan} 7$ combination while the lowest value was obtained from untreated vine of the 41B/Sultan 7 combination. Moreover there were significant treatment $\mathrm{x}$ rootstock interactions for the both Manisa Sultaniand Sultan 7 varieties in terms of dry root weight. The highest $(10.93 \mathrm{~g})$ and lowest value $(4.06 \mathrm{~g})$ was observed in hot water treated vines and untreated vines of $41 \mathrm{~B} / \mathrm{Sultan} 7$ combination.

According to the fresh and dry weights of the shoots and roots, generally hot water treatments had positive effects except 5BB x variety combinations. It was observed that $5 \mathrm{BB}$ rootstock $\mathrm{x}$ variety combination was the most sensitive combination in this experiment. On the other hand there were not determined any adverse effects of thermotherapy treatments on bud and tissue vitality. Also similar results were found in previous studies $[3,7,17,22$, 23].

\section{Conclusion}

Thermotherapy treatment at $50^{\circ} \mathrm{C}$ for 30 minutes was found an effective method against Agrobacterium vitis in previous studies. Therefore this technique was applied to the dormant grapevine propagation materials before the grafting process in an attempt to examine the growing period of different rootstocks $x$ variety combinations.

It is known that the tolerance of plant material to the hot water treatment is an important matter. Overall the findings reveal that in this study there were no any adverse effects on bud and tissue vitality in Sultan 7 and Manisa Sultanigrafted onto 5BB, 41B and $1613 \mathrm{C}$ rootstocks. On the other hand it was observed that combination of $5 \mathrm{BB}$ rootstock with the varieties was more sensitive than the others against to the thermotherapy treatment. Therefore growers should pay attention to this relation between Kober 5BB rootstock and thermotherapy treatment.

Moreover hot water treated vines of $1613 \mathrm{C} / \mathrm{Sultan}$ 7 and 41B/Sultan 7 combinations became prominent in terms of final take. As can be seen from the results hot water treated cuttings of Sultan 7, which is a raisin variety, has stood out with its high final take values for all rootstocks.

In addition, considering that $41 \mathrm{~B}$ is a hard-rooted rootstock and final take values are generally low, it is so important that the thermotherapy has a positive effect on final take of 41B rootstock and variety combinations.

As a result, in order to grow healthy grafted vines and provide economic technique against Agrobacterium vitis 
in Sultan 7 and Manisa sultanıvarieties - newly registered varieties which were obtained from the Sultani Çekirdeksiz - thermotherapy treatment needs to be widespread.

\section{References}

[1] FAO, 2014. Accessed date: 20.04.2017

[2] C. Bauer, T.F. Schulz, D. Lorenz, K.W. Eichhorn, R. Plapp, Vitis 33, 25-29 (1994)

[3] K. Ophel and A. Kerr, Internat. Journ of Systematic and Evolutionary Microbiology 40, 236-241 (1990)

[4] T.J. Burr, and B.H. Katz, Phytopathology 73, 163-165 (1983)

[5] T. Martinson and T. Burr, Research news from Cornell's Viticulture and Enology Program, Research Focus 1 (2012)

[6] Anonymous, Official gazette, (Accessed date: 17 Ocak 2016) (2011)

[7] T.J. Burr, K. Ophel, B.H. Katz, A. Kerr, Plant Dis. 73, 242-245 (1989)

[8] C. Bazzi, E. Stefani, R. Gozzi, T.J. Burr, C.L. Moore, A. Anaclerio, Vitis 30, 177-187 (1991)

[9] T.J. Burr, C.L. Reid, D.F. Splittstoesser, M. Yoshimura, Am. J. Enol. Vitic. 47, 119-123 (1996)

[10] Caudwell, J. Larrue, E. Boundon-Padieu, G.D. Mclean. Aust. J. Grape Wine Res. 3, 21-25 (1997)
[11] C. Ilgın, and Y.Z. Gürsoy, TAGEM, 6.Türkiye Bağcılık Semp. I, 114-120 (2005)

[12] F. Halleen, P.H. Fourie, P.W. Crous, Plant Pathol. 56, 637-645 (2007)

[13] E. Kacar, B. Işçi, A. Altindişli, Bulletin de l'OIV 85, 974 (2012)

[14] D. Le Roux, Farming in South Africa, pamphlet VORI, 212 (1988)

[15] İ. Korkutal, G. Kaygusuz, S. Bayram, Afr. J. Biotech. 10, 15123-15129 (2011)

[16] N. Sivritepe and C. Türkben, Uludağ Üniv. Ziraat Fak. Derg. 15, 47-58 (2001)

[17] H. Mahmoodzadeh, A. Nazemieh, I. Majidi, I. Paygami and A. Khalighi, J. Phytopathology. 151, 481-484 (2003)

[18] İ. Yavaş and Y. Fidan, Türkiye 1. Fidancıllk Sempozyumu. Ankara. 79-84 (1991)

[19] O. Soltekin, Y. Savaş, E.T. Özcan, E. Kacar, Turkish Journal of Agricultural and Natural Science 4(1), 30-39 (2017)

[20] K. Ophel, T.J. Burr, P.A. Magarey, A. Kerr, Australasian Plant Pathol. 17, 61-6 (1988)

[21] R.L. Wample, A. Bary, T.J. Burr, Am. J. Enol. Viticult. 42, 67-72 (1991)

[22] P.G. Goussard, Vitis 16, 272-278 (1977)

[23] C.J. Orffer and P.G. Goussard, Vitis. 19, 1-3 (1980) 